\title{
NEUTROPHIL SCATTERING DATA DRIVEN PRE-MICROSCOPIC FLAGGING OF ACUTE LEUKEMIC CASES
}

\author{
Rana Zeeshan-Haider ${ }^{1,2,3 *}$, Eloisa URrechaga ${ }^{4}$, IKram Uddin-UjJan ${ }^{5}$, AND Tahir Sultan-Shamsi ${ }^{1}$ \\ ${ }^{1}$ National Institute of Blood Disease and Bone Marrow Transplantation, Karachi, Pakistan; ${ }^{2}$ Jamil-ur-Rahman Center for \\ Genome Research, International Center for Chemical and Biological Sciences, University of Karachi, Karachi, Pakistan; \\ ${ }^{3}$ Genetic Engineering and Biotechnology Institute, Marmara Research Center, TUBITAK, Gebze-Kocaeli, Turkey; \\ ${ }^{4}$ Hematology Laboratory, Hospital Galdakao-Usansolo, Galdakao, Spain; ${ }^{5}$ Basic Medical Sciences, Liaquat University of \\ Medical and Health Science, Jamshoro, Pakistan.
}

\begin{abstract}
Background: The hematology analyzer, Sysmex $\mathrm{XN}-1000$, generates white blood cell count with varying scattering intensities during a complete blood count $(\mathrm{CBC})$ analysis. Objectives: The objectives of the study were to study the predictive role of median and coefficient of variation of neutrophil scattering items in blood samples for differentiation of leukemic subjects. Methods: We evaluated six neutrophil scattering parameters: neutrophil side scatter mean intensity, neutrophil side fluorescence light (SFL) mean intensity, neutrophil forward scatter mean intensity, neutrophil side scatter area distribution width (NE-WX), neutrophil SFL area distribution width (NE-WY), and neutrophil forward scatter area distribution width (NE-WZ), measured in white blood cell differential scattergram generated by the hematology analyzer (Sysmex XN-1000) at an academic medical center. Results: We collected 433 blood samples from acute myeloid leukemia (AML) and acute lymphoid leukemia (ALL) cases and normal controls. AML group showed highly significant differences in the mean values compared with the control group. Out of six neutrophil scattering items, NE-WX, NE-WY, and NE-WZ showed high efficiency, with area under the curve (AUC) values of $0.764,0.748$, and 0.757 , respectively, to differentiate $A M L$ from ALL cases and control groups. When comparing combined acute leukemia cases (AML plus ALL) with the control group, NE-WX, NE-WY, and NE-WZ generated highly significant AUC values $(0.840,0.884$, and 0.801 , respectively). Conclusion: The neutrophil scattering parameters generated during $C B C$ analysis provide a new tool for the prediction of acute leukemia and its lineage. (REV INVEST CLIN. 2020;72(1):37-45)
\end{abstract}

Key words: Acute leukemia. Sysmex. Hematology analyzer. Neutrophil scattering items.

\section{INTRODUCTION}

Acute leukemias represent a heterogeneous group of blood cancers with uncontrolled proliferation of immature and/or abnormal hematopoietic cells without any relationship with a specific age group, although there is a predominance of myeloid neoplasms in adults while lymphoblastic malignancies are more common in children. To classify acute leukemia, a complex approach is followed, including
*Corresponding author:

Rana Zeeshan Haider

E-mail: zeeshan3335@yahoo.com
Received for publication: 25-07-2019

Approved for publication: 19-09-2019

DOI: $10.24875 / R I C .19003194$

0034-8376 / (c) 2019 Revista de Investigación Clínica. Published by Permanyer. This is an open access article under the CC BY-NC-ND license (http://creativecommons.org/licenses/by-nc-nd/4.0/). 
various laboratory techniques: peripheral blood and bone marrow morphological examination, immunophenotyping by flow cytometry and/or immunohistochemistry, and identification of specific molecular and cytogenetic abnormalities. Cell morphology is usually the first and one of the most important diagnostic parameters generated by the hematology laboratory. Nevertheless, over the past decades, due to increasing economic pressures and workload and with the progress in automated hematology analyzers for blood cell counting and differential, the practice of peripheral morphologic examination steadily decreased. Moreover, cytochemistry and later flow cytometry largely replaced morphologic examination. The presence or absence of abnormal cells requires a trained hematologist/pathologist to examine meticulously the slide under a microscope and reports the findings. This generally takes $2-4 \mathrm{~h}$ or more before the clinician obtains information about a suspected case of acute leukemia. When patients with acute leukemia present with very high white blood cell (WBC) count, it is easier to suspect it; however, when the WBC is normal or low, the index of suspicion for the presence of blasts is rather low and examination of peripheral blood count is usually not employed, thereby delaying the diagnosis. Along the above-said high counts and abnormalities, abnormal maturation of neutrophils on both ends (nuclear [such as abnormal nuclear segmentations, Auer rods], and cytoplasmic side [such as a defect in primary and/or secondary granule's constituents]) is also noted in acute leukemia, especially in acute myeloid leukemia (AML), which strengthens the suspicion of acute leukemia ${ }^{1-3}$.

The modern hematology analyzers flag the abnormal complete blood count ( $C B C$ ) analysis and prompt the technologist to prepare the peripheral blood film for validation of the flagged finding. Sysmex $\mathrm{XN}$ series $C B C$ analyzers are flow cytometry-based instruments, in which the blood sample is mixed with special reagents (surfactants) that cause the hemolysis of red blood cells (RBC) and platelets and facilitate the penetration of the reagent (into the WBCs) used in the following step. Then, a specific reagent containing a fluorescence dye (Polymethine dye) is used which stains the cell's nucleic acid content either in the nucleus or organelles such as mitochondria and rough endoplasmic reticulum ${ }^{4}$. A laser light of $633 \mathrm{~nm}$ is used to hit the cells flowing in a line by a stream of fluid and sequentially irradiated by a laser. Flowing of cells in light causes their scattering at various angles and also the emission of fluorescence light from the fluorescent dye, both of which are measured and processed for the generation of a 2D scatterplot on the main screen of the analyzer (Fig. 1). In addition, the values for these measurements can be viewed in background data under different titles as WBC or white blood cell differential (WDF) channel research parameters. The value of forward scattering light (FSC) and side-scattering light (SSC) are proportional to the size and internal complexity of the cell, respectively. In addition, the intensity of side fluorescence light (SFL) reflects the cell's DNA/RNA content ${ }^{5}$.

In this study, to focus the abnormal maturation of neutrophils in a quantitative manner for the prediction of acute leukemia, we selected the values (parameters) of FSC, SSC, and SFL for neutrophils: neutrophils complexity (neutrophil side scatter mean intensity [NE-SSC] ) and the width of dispersion (area) of neutrophils complexity (neutrophil side scatter area distribution width $[N E-W X]$ ), neutrophils fluorescence intensity (neutrophil side fluorescence light mean intensity [NE-SFL]) and width of dispersion (area) of neutrophils fluorescence (neutrophil side fluorescence light area distribution width [NE$W Y]$ ), and neutrophils size (neutrophil forward scatter mean intensity [NE-FSC]) and width of dispersion (area) of neutrophils size (neutrophil forward scatter area distribution width [NE-WZ]). The value of NESSC, NE-SFL, NE-FSC, NE-WX, NE-WY, and NE-WZ varies in healthy and ill population and can be investigated as an important predictive marker in different hematological malignancies. There are some emerging data on the utilization of these non-reportable research parameters in sepsis ${ }^{6-10}$, myelodysplastic syndrome ${ }^{11-15}$, and severe infections ${ }^{16-19}$. Studies concluded that such biomarkers might provide useful information for the early diagnosis and adequate management of these disorders. These parameters have been used in acute and chronic leukemia, but they have not been extensively studied especially in big data sets. The ability of Sysmex $\mathrm{XN}-1000$ to generate $W B C$ scattering parameters during a routine $C B C$ analysis can be used to identify leukemic samples and thus minimize the delay in the diagnosis of such cases. The objective of this study was to evaluate the potential of NE-SSC, NE-SFL, NE-FSC, 
Figure 1. Position of different cell populations on white blood cell differential scatterplot by Sysmex XN-1000 hematology analyzer, represented by oblong colored areas. X-axis shows side scatter (SSC) of laser light, Y-axis represents side fluorescence (SFL) and forward scatter (FSC). Through different colors, clusters of various types of leukocytes are represented: light blue for neutrophils, pink indicates lymphocytes, monocytes by green, and red for eosinophils. (A) Neutrophil scattering parameters are shown by arrow for granularity (SSC) on X-axis: NE-X and neutrophil side scatter area distribution width, while pulse neutrophil fluorescence light intensity (SFL) on Y-axis: NE-Y and neutrophil side scatter area distribution width. (B) Scattering items for size of neutrophils are presented with arrow on $\mathrm{X}$-axis: NE-Z and neutrophil forward scatter area distribution width.

A

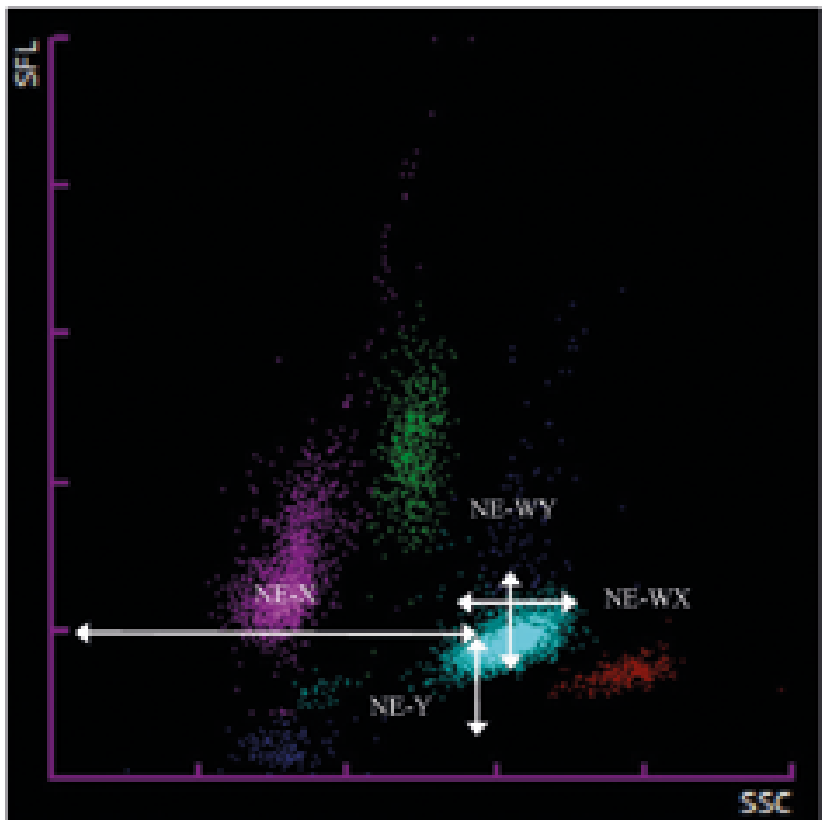

NE-WX, NE-WY, and NE-WZ to predict acute leukemia blood samples.

\section{METHODS}

\section{Subjects and procedures}

Study subjects were individuals suspected of having acute leukemia presenting to the hematology outpatient clinic of an academic research center (NIBD and BMT) in Karachi, Pakistan, for 18 months. Healthy blood donors were included as control subjects. The Institutional Ethics Committee approved the study and informed consent was obtained from study subjects. Clinical and demographic information of patients and controls was recorded.

Blood samples were collected in $\mathrm{K}_{3}$ EDTA plastic tubes (Becton Dickinson, USA) and analyzed on XN-1000 (Sysmex Co., Kobe, Japan) within an hour of sample
B

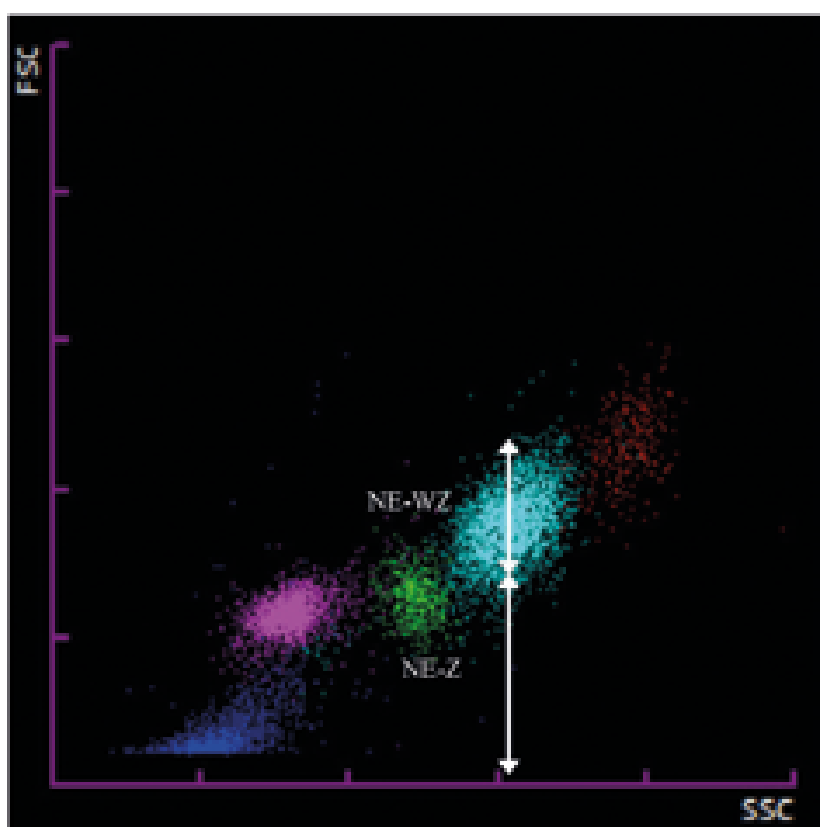

collection. The final diagnosis of acute myeloid (AML) and acute lymphoblastic (acute lymphocytic leukemia [ALL]) leukemia was made based on peripheral blood film, bone marrow examination, confirmed through immunophenotyping (by flow cytometry or immunohistochemistry), cytogenetics, and/or molecular genetics. Non-leukemic patients and those with a history of chemotherapy and blood transfusion were excluded from the study. Acute leukemia cases having > 1000 WBC count and at least 5\% immature/ abnormal WBCs cell count in peripheral blood with complete diagnostic workup were included in this study. Data on standard CBC parameters were recorded. The values of NE-SSC, NE-SFL, NE-FSC, NEWX, NE-WY, and NE-WZ were collected.

\section{Statistical analysis}

SPSS 20.0 was used for the analysis of data and $p<0.05$ was considered significant. Calculations of mean, standard deviation (SD), and significance 
Table 1. Mean, SD, and significance (p-) values for classical CBC items provided by CBC (Sysmex XN-1000) analyzers, separated by study groups

\begin{tabular}{|c|c|c|c|c|}
\hline Parameters & $\begin{array}{c}\text { Control } \\
(\text { Mean } \pm \text { SD })\end{array}$ & $\begin{array}{c}\text { AML } \\
(\text { Mean } \pm \text { SD })\end{array}$ & $\begin{array}{c}\text { ALL } \\
(\text { Mean } \pm S D)\end{array}$ & $\begin{array}{c}\text { p-value } \\
\text { (AML vs. } A L L)\end{array}$ \\
\hline $\mathrm{Hb}$ & $13.91 \pm 1.25$ & $7.58 \pm 2.60^{*}$ & $5.85 \pm 3.46^{*}$ & $<0.001$ \\
\hline $\mathrm{RBC}\left(10^{12} / \mathrm{L}\right)$ & $4.85 \pm 0.43$ & $3.53 \pm 2.35^{*}$ & $5.29 \pm 3.00^{*}$ & $<0.001$ \\
\hline PCV & $42.64 \pm 3.49$ & $25.19 \pm 7.61^{*}$ & $24.73 \pm 7.63^{*}$ & 0.057 \\
\hline MCV & $88.04 \pm 3.27$ & $89.92 \pm 10.06^{*}$ & $83.93 \pm 9.12^{*}$ & 0.388 \\
\hline $\mathrm{MCH}$ & $28.71 \pm 1.09$ & $29.32 \pm 3.43^{*}$ & $27.35 \pm 2.91^{*}$ & 0.072 \\
\hline $\mathrm{MCHC}$ & $32.63 \pm 1.19$ & $32.47 \pm 2.02^{*}$ & $32.63 \pm 1.92^{*}$ & 0.766 \\
\hline WBC $\left(10^{9} / \mathrm{L}\right)$ & $7.63 \pm 1.25$ & $37.15 \pm 63.58^{*}$ & $70.91 \pm 107.47^{*}$ & $<0.001$ \\
\hline $\operatorname{PLT}\left(10^{3} / \mu \mathrm{L}\right)$ & $278.70 \pm 53.60$ & $59.48 \pm 83.57^{*}$ & $53.74 \pm 62.92^{*}$ & 0.684 \\
\hline NEUT\# $\left(10^{3} / \mu \mathrm{L}\right)$ & $4.35 \pm 0.93$ & $9.73 \pm 27.80$ & $3.24 \pm 4.22^{*}$ & 0.350 \\
\hline LYMPH\# $\left(10^{3} / \mu \mathrm{L}\right)$ & $2.45 \pm 0.54$ & $8.24 \pm 12.67^{*}$ & $47.75 \pm 77.40^{*}$ & $<0.001$ \\
\hline MONO\# $\left(10^{3} / \mu \mathrm{L}\right)$ & $0.53 \pm 0.11$ & $19.51 \pm 40.24^{*}$ & $20.08 \pm 42.25^{*}$ & 0.031 \\
\hline $\mathrm{EO} \#\left(10^{3} / \mu \mathrm{L}\right)$ & $0.23 \pm 0.14$ & $0.16 \pm 0.89 *$ & $0.13 \pm 0.28^{*}$ & 0.581 \\
\hline BASO\# $\left(10^{3} / \mu \mathrm{L}\right)$ & $0.04 \pm 0.01$ & $0.71 \pm 0.19^{*}$ & $0.15 \pm 0.38^{*}$ & $<0.001$ \\
\hline NEUT\% (\%) & $56.92 \pm 6.03$ & $24.80 \pm 20.59^{*}$ & $13.11 \pm 17.23^{*}$ & $<0.001$ \\
\hline LYMPH\% (\%) & $32.28 \pm 5.72$ & $37.89 \pm 23.12$ & $64.35 \pm 22.06^{*}$ & $<0.001$ \\
\hline MONO\% (\%) & $7.09 \pm 1.15$ & $36.45 \pm 23.83^{*}$ & $21.06 \pm 18.03^{*}$ & $<0.001$ \\
\hline $\mathrm{EO} \%(\%)$ & $3.14 \pm 1.78$ & $0.67 \pm 1.97^{*}$ & $0.49 \pm 0.95^{*}$ & $<0.001$ \\
\hline BASO\% (\%) & $0.55 \pm 0.25$ & $0.17 \pm 0.32 *$ & $0.22 \pm 0.29 *$ & 0.007 \\
\hline IG\# $\left(10^{3} / \mu \mathrm{L}\right)$ & $0.26 \pm 0.01$ & $1.79 \pm 4.63^{*}$ & $0.73 \pm 1.50^{*}$ & $<0.001$ \\
\hline IG\% (\%) & $0.34 \pm 0.19$ & $4.52 \pm 6.61^{*}$ & $1.76 \pm 2.93^{*}$ & $<0.001$ \\
\hline
\end{tabular}

${ }^{*}$ p-value < 0.05 against control. CBC: complete blood count; SD: standard deviation; ALL: Acute Lymphocytic Leukemia; AML: Acute Myeloid Leukemia; RBC: red blood cell; IG: immature granulocyte.

$(p-)$ values against control and between acute leukemia groups were carried out using SPSS. To determine the predictive ability of neutrophil scattering items for acute leukemia, receiver operating characteristic (ROC) curve and the area under the curve (AUC) were generated.

\section{RESULTS}

A total of 433 subjects were analyzed. Of these, 76 were diagnosed as ALL, 82 as AML, and 275 were healthy subjects (control group). The control-to-patient group (ALL and $A M L$ ) ratio was 1.76. The mean ages of patients and controls were $32.76 \pm 19.9$ years and $29 \pm 11.5$, respectively. The male-to-female ratios in patients and control group were 1.2 and 1.3 , respectively. Values of mean, SD, and significance ( $p$-value) for classical CBC parameters among study groups are presented in table 1 , and those for neutrophil scattering items generated by the Sysmex XN-1000 hematology analyzer are presented in table 2 . Significance ( $p$-value) was calculated and presented in two separate manners: (1) for each disease group (AML or ALL) compared with the control group and symbolized by "*" if found significant; and (2) between disease groups (AML vs. ALL), which is shown in the last column of tables 1 and 2 .

\section{Differentiation between disease group (AML or ALL) and healthy control group}

A significant increase was observed in several classical (routine) and neutrophil scattering $C B C$ items in both $A M L$ and $A L L$ compared to healthy controls 
Table 2. Mean, SD, and significance (p-) values for six neutrophil scattering parameters provided by CBC (Sysmex $\mathrm{XN}-1000$ ) analyzers, separated by study groups

\begin{tabular}{|c|c|c|c|c|}
\hline Parameters & $\begin{array}{c}\text { Control } \\
(\text { Mean } \pm \text { SD })\end{array}$ & $\begin{array}{c}\text { AML } \\
(\text { Mean } \pm \text { SD })\end{array}$ & $\begin{array}{c}A L L \\
(\text { Mean } \pm \text { SD) }\end{array}$ & $\begin{array}{c}\text { p-value } \\
\text { (AML vs. ALL) }\end{array}$ \\
\hline$[\mathrm{NE}-\mathrm{SSC}(\mathrm{ch})]$ & $149.17 \pm 5.55$ & $141.24 \pm 13.50^{*}$ & $149.64 \pm 9.25^{*}$ & $<0.001$ \\
\hline [NE-SFL(ch)] & $46.98 \pm 2.21$ & $54.32 \pm 19.33^{*}$ & $50.71 \pm 8.30^{*}$ & 0.033 \\
\hline$[N E-F S C(c h)]$ & $86.95 \pm 3.91$ & $72.35 \pm 11.25^{*}$ & $80.89 \pm 7.03^{*}$ & 0.505 \\
\hline$[N E-W X]$ & $304.64 \pm 17.13$ & $432.54 \pm 125.52^{*}$ & $386.73 \pm 108.57^{*}$ & 0.699 \\
\hline$[N E-W Y]$ & $609.48 \pm 26.96$ & $1364.63 \pm 769.58^{*}$ & $1226.47 \pm 616.40^{*}$ & 0.881 \\
\hline$[N E-W Z]$ & $610.18 \pm 42.60$ & $820.95 \pm 249.46^{*}$ & $721.08 \pm 203.64^{*}$ & $<0.001$ \\
\hline
\end{tabular}

${ }^{*}$ p-value < 0.05 against Control. ALL: Acute Lymphocytic Leukemia; AML: Acute Myeloid Leukemia; SD: standard deviation; NE-SFL: neutrophil side fluorescence light mean intensity; NE-FSC: neutrophil forward scatter mean intensity; NE-SSC: neutrophil side scatter mean intensity; NE-WX: neutrophil side scatter area distribution width; NE-WY: neutrophil side fluorescence light area distribution width; NE-WZ: neutrophil forward scatter area distribution width.

(Table 1). Patients had significantly higher rates of leukocytes, lymphocytes, and monocytes in both types of acute leukemia; hemoglobin and platelet counts were decreased while neutrophil count was not significantly modified. A significant difference was also noted between disease and reference (control) groups, whatever the cause, for these neutrophil scattering items (Table 2): NE-SFL, NE-WX, NEWY, and NE-WZ were increased, while NE-SSC and NE-FSC were slightly decreased or found near normal ranges.

Both AML and ALL disease groups mainly followed the same trend in routine CBC items, except for the lymphocyte percent count, which remained unmodified for AML. Interestingly, four neutrophil scattering items, NE-SFL, NE-WX, NE-WY, and NE-WZ, had higher values, and NE-FSC had lower values (against control for $A M L$ ) compared to ALL, while exceptionally NE-SSC remained unaffected in ALL patients.

\section{Differentiation between AML and ALL}

Considering the whole population of our disease data set, from classical $C B C$ items, the level of hemoglobin and counts for RBCs, WBCs, absolute lymphocyte, absolute basophil, percent neutrophil, lymphocyte, monocyte, and eosinophil, absolute, and percent immature granulocyte (IG) were found to be significantly different when comparing AML to ALL (Table 1). Leukocytes, absolute, and percent lymphocytes were significantly higher in ALL $(p<0.0001)$, while for
AML, percent monocyte, absolute, and percent IG were found with significantly increased counts.

As shown in table 2, Sysmex $\mathrm{XN}-1000$ analyzer provided discriminating values for only NE-SSC and NEWZ between AML and ALL.

\section{ROC curves}

Neutrophil scattering items were used to establish ROC curves (Fig. 2), differentiating between disease group and healthy controls (Fig. 2: AL, AML, and NonAML [ALL plus control]); AML, and ALL and Non-ALL (AML plus control). In general, three out of six neutrophil scattering parameters (NE-WX, NW-WY, and NE-WZ) provided significant discrimination with higher AUC values. In differentiating acute leukemia (disease) groups from non-disease (control) group, excellent discriminating power as $A \cup C$ values were achieved for NE-WX, NE-WY, and NE-WZ: 0.840, 0.884 and 0.801 , respectively (Fig. 2). For disease groups, lesssignificant AUC values of $0.601,0.680$, and 0.558 for NE-WX, NE-WY, and NE-WZ, respectively, in ALL cases were found, while for AML, NE-WX, NE-WY, and NE-WZ provided significant AUC values of 0.764 , 0.748 , and 0.757 , respectively.

\section{Increase in width and shifting of neutrophil's scattering area in disease group}

The light-scattering intensities are processed and presented on the main screen of the analyzer as 
Figure 2. Receiver operating characteristic curves and area under the curve values of neutrophil scattering items, neutrophil side scatter area distribution width, neutrophil side fluorescence light area distribution width, and neutrophil forward scatter area distribution width provided significant results for all three types of discrimination (AL: acute leukemia from control; acute myeloid leukemia [AML] from Non-AML [acute lymphocytic leukemia (ALL) plus control]; ALL from Non-ALL [AML plus control]).
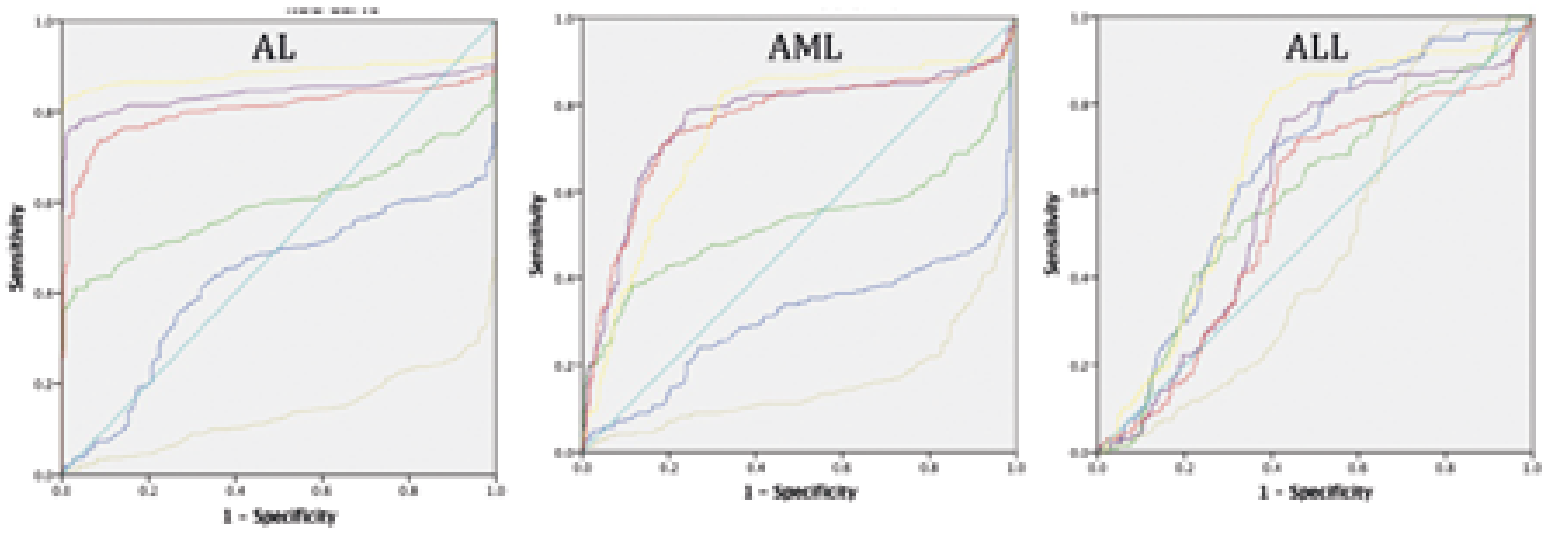

\begin{tabular}{clll}
\hline \multirow{2}{*}{ Test Result Variable(s) } & \multicolumn{3}{c}{ AUC values } \\
\cline { 2 - 4 } & AL & AML & ALL \\
\hline$[$ NE-SSC(ch)] & 0.429 & 0.310 & 0.659 \\
{$[$ NE-SFL(ch)] } & 0.596 & 0.526 & 0.593 \\
{$[$ NE-FSC(ch)] } & 0.138 & 0.159 & 0.475 \\
{$[$ NE-WX] } & 0.840 & 0.764 & 0.601 \\
{$[$ NE-WY] } & 0.884 & 0.748 & 0.680 \\
{$[$ NE-WZ] } & 0.801 & 0.757 & 0.558 \\
\hline
\end{tabular}

scatterplots. Figure 3 shows the increase in width and/or shifting of neutrophil's scattering area on WDF scatterplots in AML and ALL (by comparing white circles in AML and ALL against the control group). As noted in figure 3 , compared to healthy subjects, the increase in width of neutrophil's scattering area was significantly prominent for both of our disease groups, while shifting of neutrophil's scattering area was comparatively less perceptible. Between disease groups, AML showed a highly discriminating scattering trend for neutrophil's area compared to a moderate increase in width of scattering area in patients with ALL.

\section{DISCUSSION}

Despite the current need for extensive testing for the complete classification approach of acute leukemias, physicians need first to know the lineage of the suspected case of leukemia. Therefore, laboratory testing usually starts with morphology and flow cytometry of the patient's blood and/or bone marrow samples before advancing to the molecular and cytogenetic testing. Flow cytometry, however, in itself is not a routine test, and is typically operated during regular work hours and only feasible in limited wellequipped laboratories and hospitals due to the requirements of experienced personnel and modern instrumentation, problems, which are more pronounced in developing countries.

Among complex tests for the diagnostic workup of acute leukemias, hematology analyzers for $C B C$ analysis provide $\mathrm{WBC}$ research (scattering) parameters that have the potential to detect morphological changes in these cells. These scattering items of WBCs, especially neutrophils, have been useful in the prediction 
Figure 3. White blood cell differential scatterplots for $\mathbf{A}=$ Healthy subject (without any abnormal or immature white blood cell), $\mathbf{B}=$ Acute myeloid leukemia, and $\mathbf{C}=$ Acute lymphocytic leukemia. White circles indicate the neutrophil's scattering area.

\section{A Control}
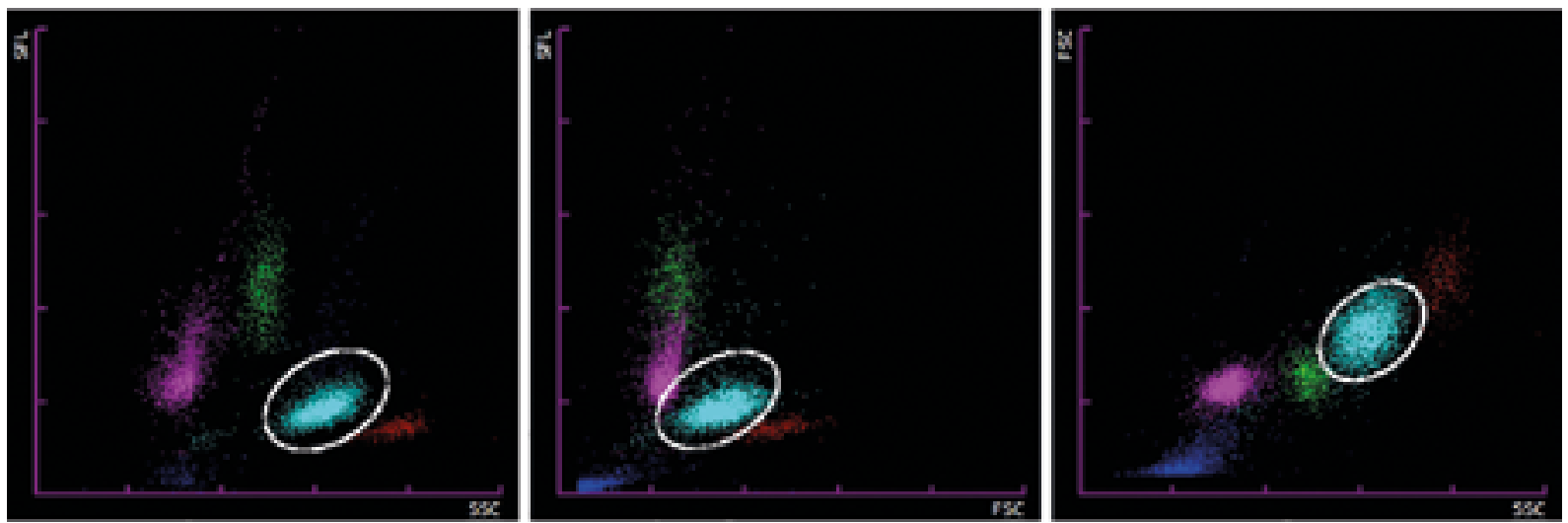

\section{B AML}
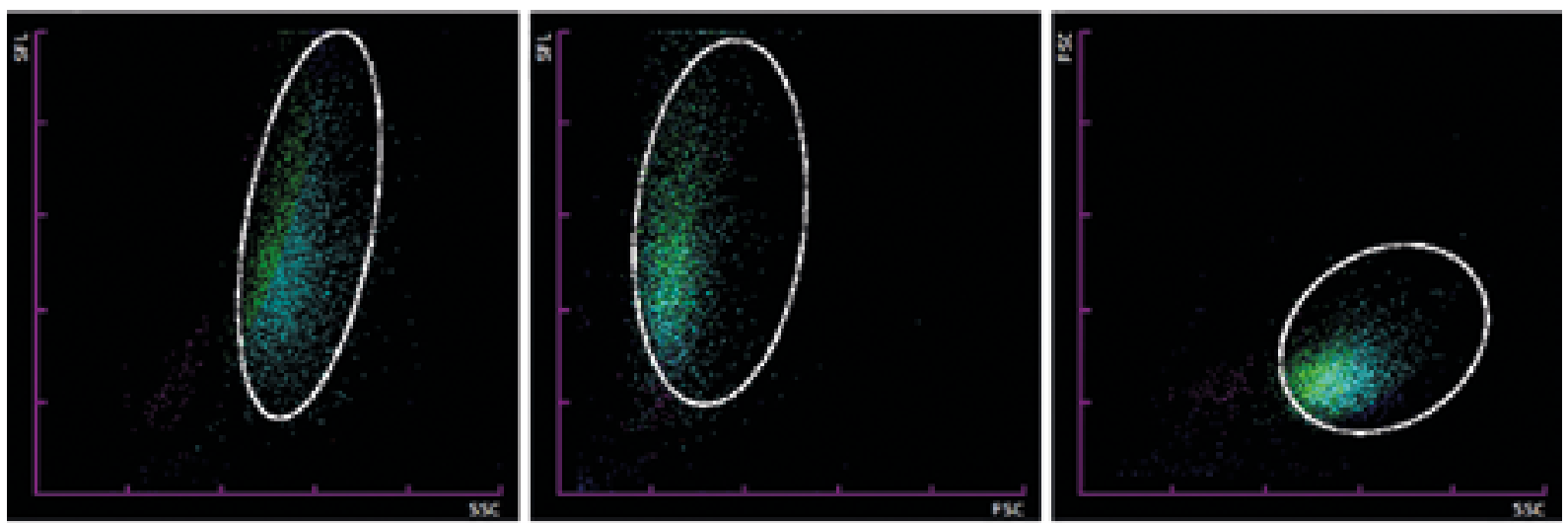

C ALL
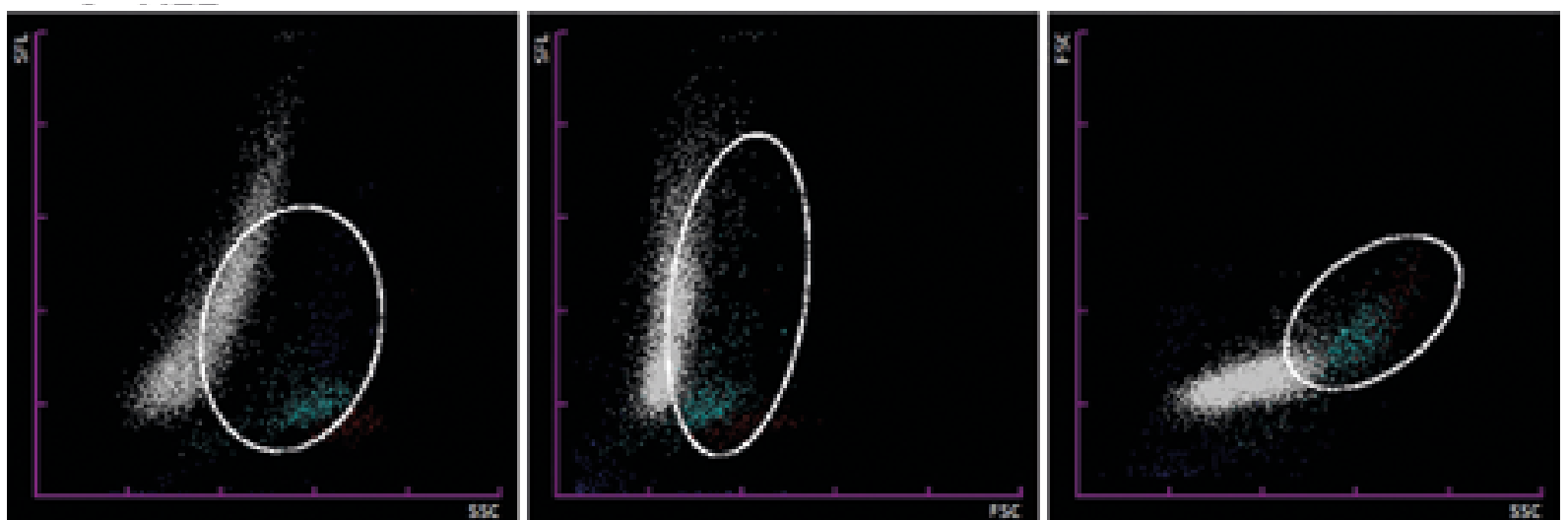

of sepsis and neutrophil dysplasia9,11,14,20. Their usefulness was not much studied for the differentiation of normal samples in comparison with immature neutrophils, including blast cell populations, in distinguishing various types of leukemic disorders or for their detection against leukemoid reaction and infections. 
A study by Yang et al. evaluated white cell scattering items for differentiation of acute leukemia lineage using Coulter $\mathrm{D} \times \mathrm{H} 800$ analyzer. In this study, the authors derived a model based on 21 items and reported very high (100\%) specificity and sensitivity for differentiation of acute promyelocytic leukemia (APL) cases, while for ALL, comparably less significant specificity and sensitivity were achieved ${ }^{21}$. Another study by Virk et al. reported the clinical utility of the white cell scattering items, scattergrams, and flags for screening of AML cases with significant specificity 22 .

The findings from our study are promising for acute leukemia since our neutrophil scattering items were not only able to detect the presence of acute leukemia but also predict its lineage (AML or ALL). Of particular importance was the performance of NE-WX, NE-WY, and NE-WZ, which was found with significantly higher values for AML followed by ALL compared with healthy subjects. Most immature cells in AML and ALL, especially APL cells, have been found to be counted near or in the neutrophil population area, which caused shifting and particularly increase in width of the neutrophil's scattering area (Fig. 3) 3 23. $^{23}$. Consequently, this area in AML cases would be a mixture of AML immature/abnormal cells and neutrophils, thus generating higher values for NE-WX, NE-Y, and NE-WZ.

A number of studies have reported the key importance of WBC morphology in the diagnosis of hematological diseases. While the microscopical review of every $C B C$ differential is not feasible, especially in labs with heavy workload, labs have now built their knowledge and expertise for the use of modern hematology analyzers to assess these morphologic characteristics in a fast, inexpensive, automated, and quantitative manner. In addition to obtaining a straightforward interpretation of results and numerical decision rules of the quantitative analysis, a screening algorithm can be implemented using the criteria of the scattering items based on big data sets. Aiming for early prediction of acute leukemia cases and its sub-classification in AML or ALL, the present study showed a potential clinical utility of these scattering items.

This study has some limitations. First, the effect of sepsis in study cases was not excluded by any reference method. However, as reported in some studies ${ }^{9,20}$, sepsis caused only a moderate increase in values of neutrophil research items, particularly parameters related with neutrophil's area width (NE-WX, NE-WY, and NE-WZ), compared against discriminatively higher values in patients with acute leukemia (where immature/abnormal WBC counted in neutrophil scattering area produced very high values). Second, any changes in research parameters based on the disease subgroup at the time of diagnosis of acute leukemia could not be evaluated in the present study. The investigation of these research (extended) parameters in differentiation and identification of subgroups of acute leukemia can provide important information, such as the prediction power and detection sensitivity of these research items for subtypes of acute leukemia, and therefore, should be addressed in the future.

In summary, we here propose a new solution for the screening of acute leukemia cases and differentiating their lineage on neutrophil scattering parameters generated along regular CBC-diff. These findings may be of clinical importance in early, cost-effective, and more accurate diagnosis (by avoiding unnecessary tests) of acute leukemia patients, especially in resource-constrained setups.

\section{REFERENCES}

1. Abdul-Hamid G. Classification of Acute Leukemia. Acute Leukemia-The Scientist's Perspective and Challenge. Rijeka: IntechOpen; 2011.

2. Davey FR, Erber WN, Gatter KC, Mason DY. Abnormal neutrophils in acute myeloid leukemia and myelodysplastic syndrome. Hum Pathol. 1988;19:454-9.

3. Gudgeon CJ, Harrington KH, Laszlo GS, Alonzo TA, Gerbing RB, Gamis AS, et al. High expression of neutrophil elastase predicts improved survival in pediatric acute myeloid leukemia: a report from the children's oncology group. Leuk Lymphoma. 2013; 54:202-4.

4. Sysmex XN Series AsG. Automated Hematology Analyzer, XN series, Administrator's Guide. Kobe, Japan: Sysmex Corporation; 2012. p. 1-132.

5. Europe S. Analyzer and Software Training: Sysmex; 2016. Available from: https://www.sysmex.com/US/en/Education/Pages/ Analyzer-and-Software-Training.

6. Arneth BM, Ragaller M, Hommel K, Tiebel O, Menschikowski M, Siegert G, et al. Novel parameters of extended complete blood cell count under fluorescence flow cytometry in patients with sepsis. J Clin Lab Anal. 2014;28:130-5.

7. Buoro S, Mecca T, Azzarà G, Apassiti Esposito S, Seghezzi M, Vavassori $M$, et al. Extended leukocyte differential count and $C$-reactive protein in septic patients with liver impairment: diagnostic approach to evaluate sepsis in intensive care unit. Ann Transl Med. 2015;3:244.

8. Luo $\mathrm{Y}$, Lin J, Chen H, Zhang J, Peng S, Kuang M, et al. Utility of neut- $X$, neut- $Y$ and neut- $Z$ parameters for rapidly assessing sepsis in tumor patients. Clin Chim Acta. 2013;422:5-9.

9. Park SH, Park CJ, Lee BR, Nam KS, Kim MJ, Han MY, et al. Sepsis affects most routine and cell population data (CPD) obtained 
using the sysmex XN-2000 blood cell analyzer: neutrophil-related CPD NE-SFL and NE-WY provide useful information for detecting sepsis. Int J Lab Hematol. 2015;37:190-8.

10. Urrechaga E, Bóveda O, Aguirre U. Role of leucocytes cell population data in the early detection of sepsis. J Clin Pathol. 2018; 71:259-66.

11. Furundarena JR, Araiz M, Uranga M, Sainz MR, Agirre A, Trassorras $M$, et al. The utility of the sysmex XE-2100 analyzer's NEUT-X and NEUT-Y parameters for detecting neutrophil dysplasia in myelodysplastic syndromes. Int J Lab Hematol. 2010; 32:360-6.

12. Haschke-Becher E, Vockenhuber M, Niedetzky P, Totzke U, Gabriel C. A new high-throughput screening method for the detection of chronic lymphatic leukemia and myelodysplastic syndrome. Clin Chem Lab Med. 2008;46:85-8.

13. Inaba T, Yuki Y, Yuasa S, Fujita N, Yoshitomi K, Kamisako T, et al. Clinical utility of the neutrophil distribution pattern obtained using the CELL-DYN SAPPHIRE hematology analyzer for the diagnosis of myelodysplastic syndrome. Int J Hematol. 2011;94:169-77.

14. Le Roux G, Vlad A, Eclache V, Malanquin C, Collon JF, Gantier M, et al. Routine diagnostic procedures of myelodysplastic syndromes: value of a structural blood cell parameter (NEUT-X) determined by the sysmex XE-2100 ${ }^{\mathrm{TM}}$. Int J Lab Hematol. 2010;32:e237-43.

15. Raess PW, van de Geijn GJ, Njo TL, Klop B, Sukhachev D, Wertheim $G$, et al. Automated screening for myelodysplastic syndromes through analysis of complete blood count and cell population data parameters. Am J Hematol. 2014;89:369-74.
16. Ansari-Lari MA, Kickler TS, Borowitz MJ. Immature granulocyte measurement using the sysmex XE-2100. Relationship to infection and sepsis. Am J Clin Pathol. 2003;120:795-9.

17. Charafeddine KM, Youssef AM, Mahfouz RA, Sarieddine DS, Daher RT. Comparison of neutrophil volume distribution width to C-reactive protein and procalcitonin as a proposed new marker of acute infection. Scand J Infect Dis. 2011;43:777-84.

18. Henriot I, Launay E, Boubaya M, Cremet L, Illiaquer M, Caillon $H_{\text {, }}$ et al. New parameters on the hematology analyzer XN-10 (SysmexTM) allow to distinguish childhood bacterial and viral infections. Int J Lab Hematol. 2017;39:14-20.

19. Jung YJ, Kim JH, Park YJ, Kahng J, Lee H, Lee KY, et al. Evaluation of cell population data on the uniCel dxH 800 coulter cellular analysis system as a screening for viral infection in children. Int J Lab Hematol. 2012;34:283-9.

20. Buoro S, Seghezzi M, Vavassori M, Dominoni P, Apassiti Esposito S, Manenti B, et al. Clinical significance of cell population data (CPD) on sysmex XN-9000 in septic patients with our without liver impairment. Ann Transl Med. 2016;4:418.

21. Yang JH, Kim Y, Lim J, Kim M, Oh EJ, Lee HK, et al. Determination of acute leukemia lineage with new morphologic parameters available in the complete blood cell count. Ann Clin Lab Sci. 2014;44:19-26.

22. Virk H, Varma N, Naseem S, Bihana I, Sukhachev D. Utility of cell population data (VCS parameters) as a rapid screening tool for acute myeloid leukemia (AML) in resource-constrained laboratories. J Clin Lab Anal. 2019;33:e22679.

23. Jung S, Chae H, Lim J, Oh EJ, Kim Y, Park YJ, et al. Differential blast counts obtained by automated blood cell analyzers. Korean J Lab Med. 2010;30:540-6. 